\title{
Contributions of Advanced Taxi Time Calculation to Airport Operations Efficiency
}

\author{
Thomas Günther ${ }^{1}$, Matthias Hildebrandt ${ }^{2}$, and Hartmut Fricke ${ }^{3}$ \\ Technische Universität Dresden, 01069 Dresden, Germany \\ Moritz Strasser ${ }^{4}$ \\ ATRiCS Advanced Traffic Solutions GmbH \& Co. KG, 79108 Freiburg, Germany
}

\begin{abstract}
The current paper stresses the high priority of accurate taxi time calculation and suitable pre-departure management strategies in the context of Airport Collaborative Decision Making (Airport CDM) and contributes to an improved overall understanding of the associated requirements. Based on data analysis performed at Frankfurt Airport this paper compares results of static and advanced taxi time calculation. Contrarily to static calculations, where look-up tables for stand-runway-relations are used, advanced taxi time calculation takes into account the current traffic situation and calculates taxi times dynamically. The paper shows that the taxi time uncertainties when using static data lead to only $42 \%$ of flights within the accuracy of $+/-2$ minutes according to the requirements of the Airport CDM Implementation Manual for short term predictions (maximum 30 minutes before off-block). The application of advanced taxi time calculation leads to significant improvements with $66 \%$ of flights within the accuracy of $+/-2$ minutes at the moment when the aircraft leaves the stand. Hence, app. $50 \%$ more predictions are compliant with the Airport CDM requirements compared to static data. Based on these results, the paper assesses the effects of improved taxi time predictability onto airport operation efficiency with particular focus set to taxi time reductions due to minimized queuing at the departure runway. It is shown that advanced taxi time calculation contributes to a reduction of delays, fuel burn, $\mathrm{CO}_{2}$ emissions and costs for airspace users compared to the use of static look-up tables. However, it is also emphasized that the shifting of delays from the runway holding positions to the stand does not necessarily contribute to efficiency improvements. It is recommended that the pre-departure sequencing strategy must be chosen cautiously in dependence on the fuel and time costs for airlines as well the amount of departure delay that has to be absorbed.
\end{abstract}

\section{Nomenclature}

$\begin{array}{lll}\text { A-SMGCS } & = & \text { Advanced Surface Movement Guidance and Control System } \\ \text { AMAN } & = & \text { Arrival Management System } \\ \text { ATC } & = & \text { Air Traffic Control } \\ \text { CDM } & = & \text { Collaborative Decision Making } \\ \text { DMAN } & = & \text { Departure Management System } \\ \text { EXOT } & = & \text { Estimated Taxi Out Time } \\ \text { RWY } & = & \text { Runway } \\ \text { SMAN } & = & \text { Surface Management System } \\ \text { TMAN } & = & \text { Turnaround Management System } \\ \text { TOBT } & = & \text { Target Off-Block Time } \\ \text { TSAT } & = & \text { Target Start Up Approval Time } \\ \text { TTOT } & = & \text { Target Take Off Time } \\ \text { VTTC } & = & \text { Variable Taxi Time Calculation }\end{array}$

\footnotetext{
${ }^{1} \mathrm{PhD}$ candidate, Chair of Air Transport Technology and Logistics, Email: guenther@ifl.tu-dresden.de.

${ }^{2}$ Student, Chair of Air Transport Technology and Logistics, Email: matthiashildebrandt@gmx.de.

${ }^{3}$ Professor, Chair of Air Transport Technology and Logistics, Email: fricke@ifl.tu-dresden.de.

${ }^{4}$ Business analyst, ATRiCS GmbH \& Co KG, Email: moritz.strasser@atrics.com.
} 


\section{Background and Motivation}

$\mathrm{T}$ he improvement of taxi time calculation methods belongs to the key elements of the Airport Collaborative Decision Making (Airport CDM) concept. Traffic prediction using variable taxi time calculation (VTTC) is assumed to contribute to increased Air Traffic Control (ATC) slot adherence and airport operations efficiency. Especially the Pre-departure sequencing process likely relies on precise predictions of outbound taxi times with regard to its objective to determine accurate Target Take Off Times (TTOT) and Target Start Up Approval Times (TSAT) under consideration of turnaround, surface movement and departure management requirements as well as airline/airport preferences.

The manifold research activities in the field of Airport CDM and departure management require precise definitions in wording. Of particular relevance for this paper is the differentiation between the Pre-departure sequencing and Departure sequencing process. Predeparture sequencing is the third element of the Airport CDM concept (according to figure 1) and focuses on the optimisation of pushback sequences in a collaborative manner. The Airport CDM Implementation Manual ${ }^{1}$ clearly states that "the Collaborative Predeparture sequence is the order that aircraft are planned to depart from their stands (push off blocks) taking into account partners' preferences. It should not be confused with the pre-take off order where ATC organise aircraft at the holding point of a runway". The second aspect rather belongs to the Departure sequencing. It is managed during taxiing and line-up by the responsible air traffic controller. Appropriate support is primarily foreseen by surface management (SMAN) planning functions of Advanced Surface Movement Guidance and Control Systems (A-SMGCS). The ICAO A-SMGCS Manual ${ }^{2}$ emphasizes: "A-SMGCS should also provide for sequencing $[\ldots .$.$] of departing aircraft, to ensure minimum delay and$ maximum utilization of the available capacity of the aerodrome." Departure management is used as the generic term in this context since the presented processes need to be managed cooperatively. Their efficiency depends amongst others mainly on the airlines' confirmed target off-block times (TOBT), estimated taxi-out times (EXOT) and departure capacity predictions. Improving only one of them seems to be of very limited value. For instance, airlines could hardly understand why they should invest in increasing their TOBT quality whereas taxi time calculation still demonstrates high uncertainties. The current paper concentrates on taxi time calculations, but it must be stated that further research especially in the context of the turnaround process and the respective TOBT prediction is required and ongoing ${ }^{34}$ as well.
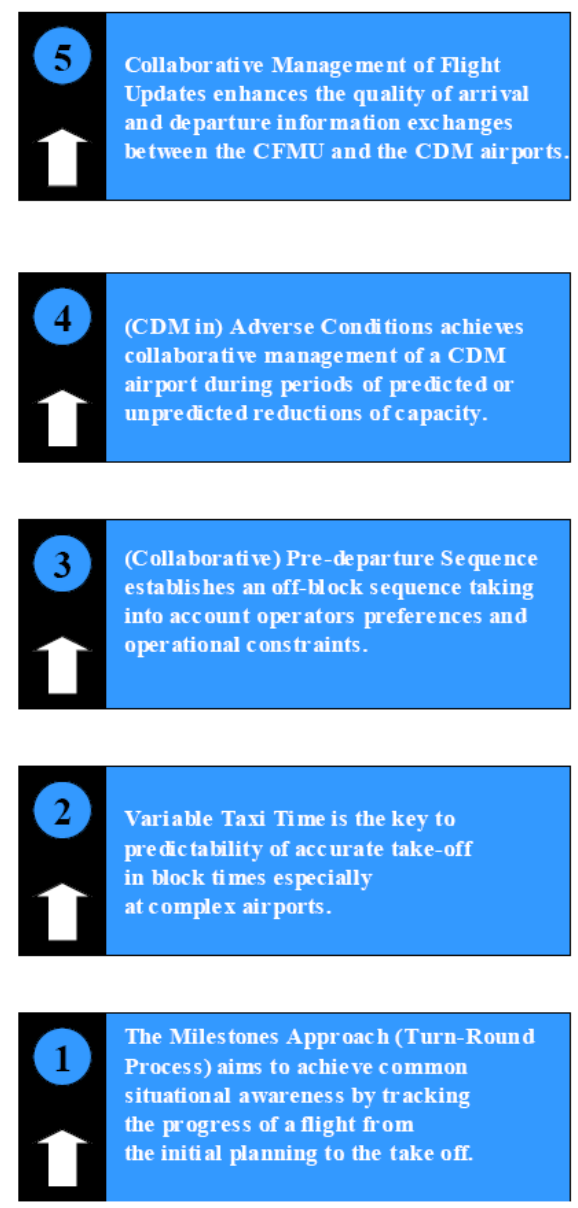

Figure 1. Airport CDM Concept

Elements [A-CDM Implementation Manual]

Taxi time is defined as the time between off-block and take-off time for outbounds respectively landing and on-block time for inbound flights. Variable taxi times are calculated either by static look-up tables or recently also dynamically. According to the Airport CDM Implementation Manual such dynamic approach is called "Advanced taxi time calculation". In order to understand the acronyms that will be used in this paper the differentiation between static and advanced can in accordance to previous studies ${ }^{5}$ be summarised at follows: Static taxi time calculation is based on statistical values, advanced taxi time calculation takes into account the planned and current surface movements and dynamically calculates holding times at intersections (and prospectively in the departure queue). Currently, taxi time calculations at airports still revert to static data. Worst case, but at several airports still applied, is the common use of a default values for all ground movements. On the other hand, VTTC includes by definition a categorisation based on the individual relations between the parking stand and the runway or vice versa. Due to a mostly wide range of taxi distances at airports, this leads to a strong reduction of uncertainties. Nevertheless, the respective analysis presented here shows that the achieved accuracy still does not comply with requirements of the Airport CDM Implementation Manual. Therein required accuracy is defined as $+/-2$ minutes for flights with maximum 30 minutes remaining time to off-block (short term prediction), $+/-5$ minutes for flights between 30 minutes and 2 hours before off-block time (medium term) and $+/-7$ minutes for flights between 2 and 3 hours before off-block time (long term). 
Taxi time calculation can contribute in two different ways to improvements in the context of departure management. First, prediction of departure delay at the holding points of the runways requires reliable information about the demand of departure (as well as arrival) traffic. Taxi time predictions enable the calculation of the arrival times at the holding points of every individual flight based on available TOBT information in combination with additional factors such as de-icing requirements. Second, the optimisation of the pre-departure sequence (TSAT allocation) can be derived from the TTOT accordingly calculated by a departure management system (DMAN) and considering local traffic situation as well as ATC slot requirements. Challenge in this context arises from the requirement to minimise delays at the holding points of the runway without negatively affecting runway throughput. This is of particular importance as some studies indicate very limited potential of a DMAN to increase runway capacity through sequence changes. Reason is the fact that the controllers obviously are able to achieve optimum separations by the skilful use of the several runway holding points; remaining space for a further optimisation by DMAN can be very small ${ }^{7}$. Accordingly, the current paper both contributes to a better understanding of appropriate TSAT allocation strategies as well as efficiency benefits to be achieved by advanced taxi time calculations.

\section{Methodology}

Investigation was performed in three steps. First, current taxi time variations were analysed based on exemplary data of outbound movements at Frankfurt Main Airport. Data were classified into 54 groups dependent on the individual apron area and the departure runway. Both average taxi times and standard variations were analysed for all groups. As static taxi time calculation reverts to the analysed average values, the according uncertainties are characterized by the same probability distribution as the taxi time distribution itself, but with an average value of zero. Hence, in order to assess average taxi time calculation uncertainties all taxi time distribution functions were shifted to an average of zero and finally weighted in accordance to the number of movements. This led to a distribution function for static taxi time uncertainties presented in chapter III.

Second, the uncertainties of advanced taxi time calculations were assessed exemplarily for Frankfurt Airport as well. Therefore, investigation reverted to the available system "SMAN Variable Taxi Time Calculation (VTTC)" ${ }^{\prime 8}$. It is applicable for taxi time calculation before and after off-block and hence designed both for system support in the context of Airport CDM as well as surface and departure management. Using the best possible estimates available for all landing and off-block times, SMAN VTTC dynamically plans for each flight a surface trajectory from which the expected taxi time is derived. To meet the recommendation of the ASMGCS manual that "surveillance information will be useful in refining the traffic planning functions associated with predicting taxi throughput and arrival/departure times" any update of flight data or operational conditions is reflected automatically in the trajectories. Forecast takes place by the simulation of all outbound and inbound movements in order to identify interactions leading to decelerations or stops and with it to increased taxi times. The determination of the taxi route depends on the given operational conditions at the airport including standard taxiways and current runways in use. Occurring conflicts are detected iteratively and resolved in accordance to the "first come first served" principle. Thereby it is distinguished into intersection conflicts, blocking conflicts and opposite route conflicts (Figure 2). During the calibration process the parameter pushback time and taxi speed have been adjusted iteratively. However, since calibration parameter were set for all flights commonly, deviations between forecast and real taxi times could only be minimised but not avoided. Remaining deviations can be considered as uncertainties of advanced taxi time calculations and are presented within the next chapter. It is clear that an inadequate parameter setting would lead to increasing uncertainties and has to be avoided.

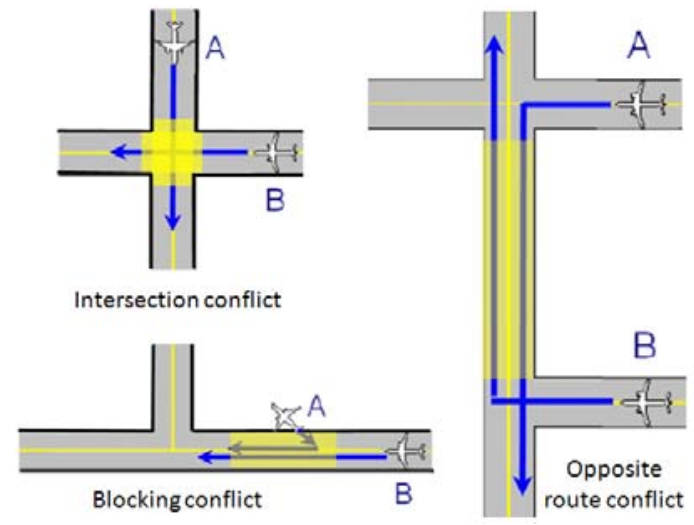

Figure 2. SMAN VTTC Conflict detection

Third, simulation was performed to assess the contributions of advanced taxi time calculation to airport operations efficiency. Therefore, a JAVA-based fast-time simulation developed at TU Dresden with a generic airport infrastructure was used. Random pushback times and taxi speeds were implemented and adjusted in accordance to the previously determined taxi time uncertainties. Traffic scenario was chosen with 103 departures (and no arrivals) scheduled within a timeframe of three hours. Departure separations were set to 120 seconds in order to build up a departure queue that is appropriable for the current investigation. Three different scenarios were performed with 10 simulation runs each: Base scenario (with no TSAT allocation), scenario 1 (TSAT allocation based on static taxi time prediction) and scenario 2 (TSAT allocation based on advanced taxi time prediction). Hence, in the base scenario the pushback sequence was not influenced by departure 
management requirements. In scenarios 1 and 2 TSAT was calculated by the formula TSAT $=$ TTOT - EXOT with TTOT determined as a result of the demand-capacity-balancing under consideration of the departure separations and according to "first come first served" principle. No ATC slot requirements were assumed. Scenarios were assessed with regard to the departure delays (both at the stand and at the runway), fuel burn and delay costs for airlines. The according results are presented in chapter IV.

\section{Taxi Time Calculation Uncertainties}

Two typical characteristics have been identified for taxi times: They do not drop below minimum values due to maximum taxi speeds, but they can reach high values because of queuing effects at the departure runway. However, it is clear that the individual minimum and average value depends on the particular relation between stand and runway (respectively runway and stand for inbounds) and the according taxi distances. Figure 3 presents the taxi time probability distribution for an exemplary stand runway relation at Frankfurt Main Airport based on the analysis of 2933 outbound movements. Average taxi time for this example is 832 seconds and the minimum time can be assumed as 360 seconds (with $99.8 \%$ of all values above). The diagram shows the significant number of very high values. As stated before, main reason is the common definition of taxi time since it includes delays arising from queuing effects at the

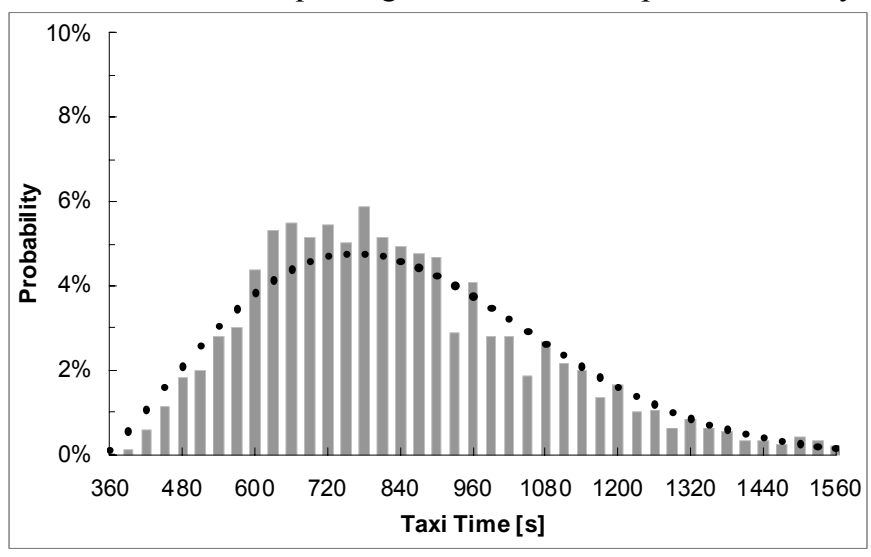

Figure 3. Empirical Probability Distribution of Taxi

Times (Frankfurt Airport, Outbound Stand A25 to RWY25R) departure runway. In times during the demand exceeding available capacity taxi times increase accordingly. Hence, taxi times can usually not be assumed as Gaussian distributed. They better fit to a Weibull distribution $\mathrm{f}(\mathrm{t})=1 / \theta^{\alpha} * \alpha^{*}\left(\mathrm{t}-\mathrm{t}_{0}\right)^{(\alpha-1) *} \mathrm{e}^{-\left[\left(\mathrm{t}-\mathrm{t}_{\mathrm{o}}\right) / \theta\right]^{\alpha}}\left(\right.$ see dotted line, in this case with $\mathrm{t}_{0}=337 \mathrm{~s}, \alpha=2.11$ and $\left.\theta=560 \mathrm{~s}\right)$.

Advanced taxi time calculation leads to Gaussian distributed uncertainties due to the individual simulation of each flight. Standard deviation for the exemplary assessment at Frankfurt Main Airport accounted for 126 seconds (average value 12 seconds). Figure 4 presents the results of the comparison between static and advanced taxi time calculation uncertainties including the illustration of the Airport CDM requirements. It is shown that actual taxi time predictability by the use of static data appears with only $42 \%$ of flights within the required accuracy of $+/-2$ minutes for short term predictions, even though prediction already covers the categorization of gate-runway relations. Analysis has also been performed with additional classifications according to wake vortex categories or surface traffic demand, but this did not lead to further improvements. Obvious reasons are stochastic effects, such as varying taxi speeds as well as pushback and taxiing conflicts, which can not completely be covered by static data. On the other hand, the application of advanced taxi time calculation leads to an increased accuracy. Taxi time predictability (at the moment when the aircraft leaves the stand) improves to $66 \%$ of flights within the accuracy of $+/-2$ minutes.

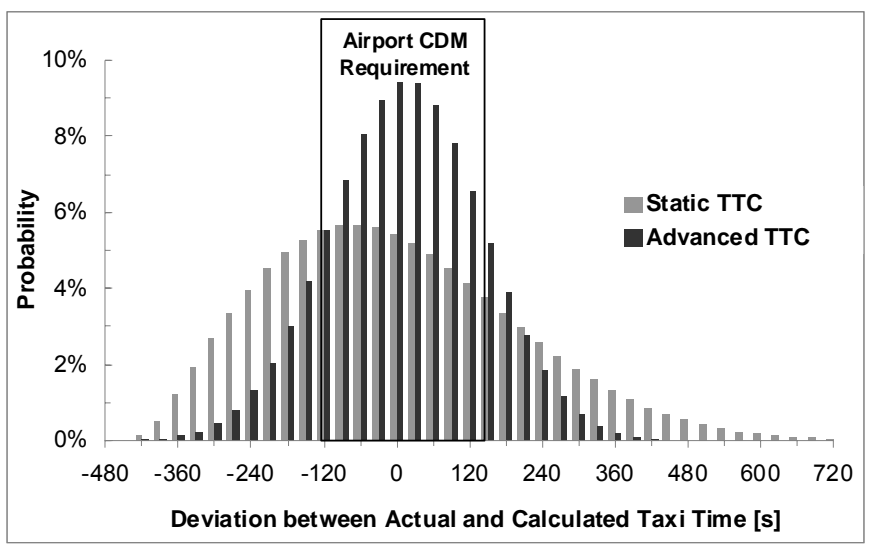

Figure 4. Comparison of Static and Advanced Taxi Time Calculation Uncertainties (Outbound at Frankfurt Airport) Hence, app. 50\% more predictions are compliant with the Airport CDM accuracy definition for short term predictions compared to static data. No results are available yet for medium term and long term predictions, but predictability will likely decrease due to higher uncertainties regarding runway and stand allocation (both for static and advanced calculation) respectively uncertainties regarding interdependencies with other traffic (only for advanced calculation).

Several strategies are expected to contribute to further improvements of data accuracy for advanced taxi time calculation. First of all, the prediction of queuing times at runways can significantly reduce uncertainties. Previous investigations proved that strong dependencies between taxi out times and the length of departure queues exist ${ }^{9}$. This goes along with the results of alternative approaches for taxi time calculation that were recently presented in the USA ${ }^{10}$ : Thereby the dynamical adaption of taxi time predictions in dependence on the 
average taxi time of the previous taxi out movements as well as the number of flights in the runway queue lead to significant improvements in taxi time predictions. Within the current investigation the queuing times are not predicted individually for every flight. Hence, according developments for advanced taxi time calculations considering departure capacity information are recommended with particular attention set to interdependencies between arrival and departure traffic.

Regarding the taxi speed prediction, individual speeds for groups of taxiways are assumed to contribute to reduced uncertainties. Taxi speeds may vary in the different areas of the airport depending on traffic complexity and air traffic control clearance necessities. Currently, default speeds were assigned to all taxiways, leading for example to deviations of taxi time accuracy in dependence on the stand and the departure runway. It is recommended to perform statistical data analysis to assess taxi speeds in dependence on suitable apron and taxiway areas. It is assumed that significant speed gradients can be noticed. Thus, different speed parameters according to the taxi routes can be set and may contribute to increased accuracy. However, this strategy could still be improved since the taxi speed further depends on the weather situation. A general trend towards lower taxi speeds during bad visibility conditions is assumed. Also towing procedures seem to influence taxi times due to their low speeds and their trend to hamper other aircraft. As one suitable solution it is recommended to dynamically adapt taxi speed for each aircraft by taking into consideration the speed of the previous segments.

\section{Effects onto Airport Operations Efficiency}

The main objectives of the pre-departure sequencing are improvements in predictability, punctuality and flexibility. With it, particular focus is set to slot adherence and consideration of airline operator requirements regarding the order in which flights can depart from their stands. Besides, also efficiency improvements can be achieved by ensuring a steadier traffic flow towards the runways in view of reduced delays at the respective holding points. According to the ICAO Global ATM Operational Concept $^{11}$ "efficiency addresses the operational and economic cost-effectiveness of gate-to-gate flight operations from a single-flight perspective". As such, in the present context efficiency mainly concerns the surface trajectory optimisation under consideration of network constraints (determined by the individual TSAT and TTOT). Hence, this paper focuses on pre-departure strategies from a single flight perspective in view of fuel and time costs. No assessment is performed yet regarding further benefits that can be achieved through off-block or takeoff sequence changes due to the ongoing developments of appropriate support systems.

Figure 5 presents the results of the performed simulation as described in chapter II. Chosen traffic scenario and the capacity limitation of 30 departures per hour led to an average queuing time at the runway holding point of approx. 600 seconds per flight (see upper diagram, base scenario). In case of shifting this delay from the runway to the stands (using the formula TSAT $=$ TTOT - EXOT) total delays increase since queuing effects at the runway can not be avoided completely due to stochastic effects. Nevertheless, queuing times at the runway can be reduced strongly with significant differences between the static and advanced taxi time calculation: Using the look up tables for taxi time calculation (scenario 1) queuing times at the runway accounted for 174 seconds per flight, by the application of advanced taxi time calculation (scenario 2) queuing times can be reduced to 132 seconds per flight. This leads to an appropriate reduction of delay related fuel burn (and with it $\mathrm{CO}_{2}$ emissions) of more than $50 \%$ (see middle diagram). Calculation was done exemplarily based on A320 fuel burn assumed by the Westminster study: $120 \mathrm{~kg} / \mathrm{min}$ APU only at stand, $630 \mathrm{~kg} / \mathrm{min}$ stationary ground at the runway (RWY) holding point. In order to assess the benefits for airspace users the total costs was analysed (lower diagram). The
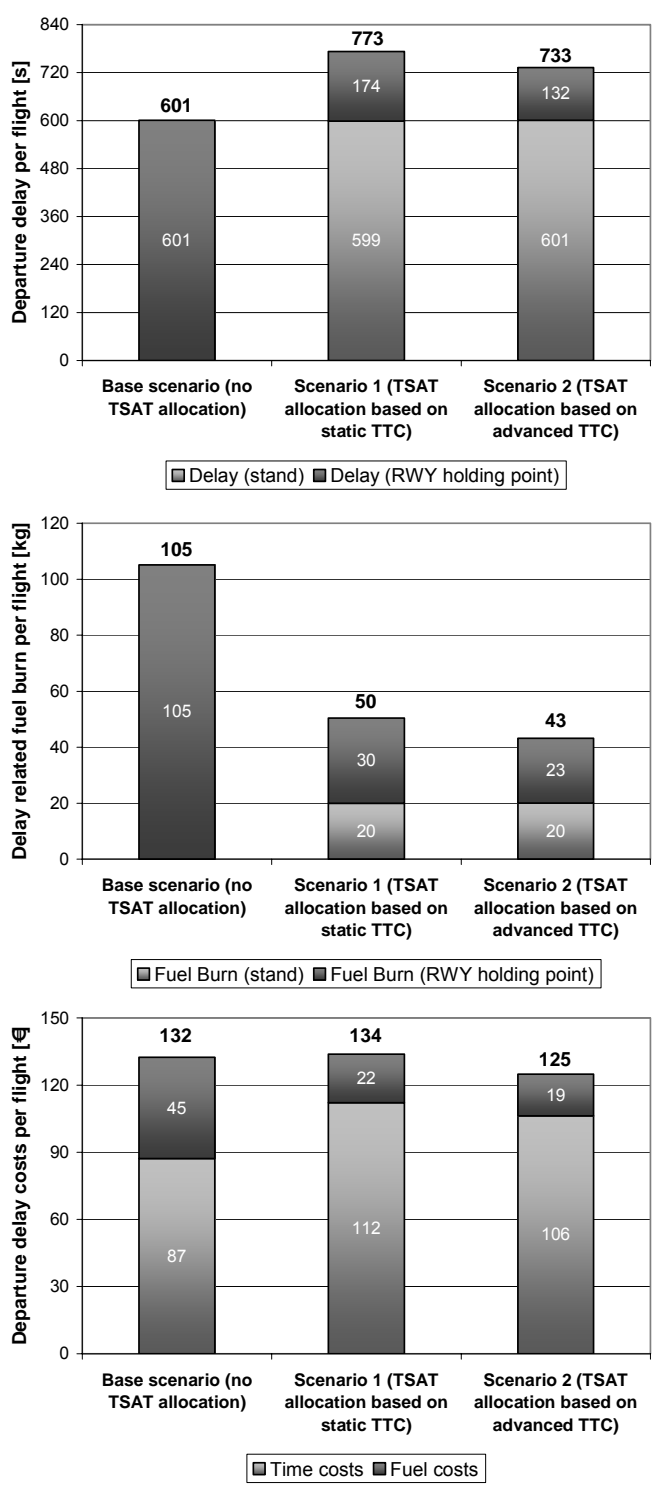

Figure 5. Impact of TSAT allocation using static or advanced taxi time calculation 
assumed fuel price of $0.43 € / \mathrm{kg}$ and delay related time costs of $8.70 € / \mathrm{min}$ are also derived from the Westminster study and correspond to a cost index (defined as the ratio between time-related costs and fuel costs) of $20 \mathrm{~kg} / \mathrm{min}$. It is shown that through TSAT allocation time costs increase but fuel costs can be reduced. In case of the static taxi time calculation this even leads to a slight increase of total costs whereas the advanced TTC enables a slight reduction of total costs.

It must be stated that results strongly depend on the cost factors and the amount of delay that has to be absorbed. For example in case of increasing time-related costs no more cost benefits for airspace users are achievable in the presented example. Hence, additional simulations were performed in view of the assessment of alternative TSAT allocation strategies. Thereby, TSAT was reduced in comparison to the previous scenarios by time buffers. Figure 6 presents the according results. First two scenarios (base, 2h) correspond to previous results, but with higher time costs of $17.40 € / \mathrm{min}$ assumed for all of the presented scenarios (corresponding to a cost index of $40 \mathrm{~kg} / \mathrm{min}$ ). Thus and contrary to the previous results, TSAT allocation without time buffers leads to higher total costs and must be considered as ineffective despite the advanced taxi time calculation. The alternative strategy in scenario $2 \mathrm{~h} 60$, where every aircraft receives a TSAT that is 60 seconds earlier compared to the origin scenario, already leads to total cost slightly below the base scenario ( $217 €$ per flight instead of $219 €$ ). Several simulations were performed leading to the minimum total cost achieved with a time buffer of 360 seconds (scenario $2 \mathrm{~h} 360$ ). With it, total delay compared to the base scenario increases by only a few seconds, but still significant fuel savings $(46 \%)$ can be achieved. Hence, total cost can be reduced to $203 €$ per flight (7\% less compared to the base scenario). Presented results show that the application of advanced taxi time calculation can contribute to efficiency benefits only in combination with suitable TSAT allocation strategies. In this case, significant improvements regarding fuel burn and $\mathrm{CO}_{2}$ emissions as well as total costs for airspace users can be achieved.
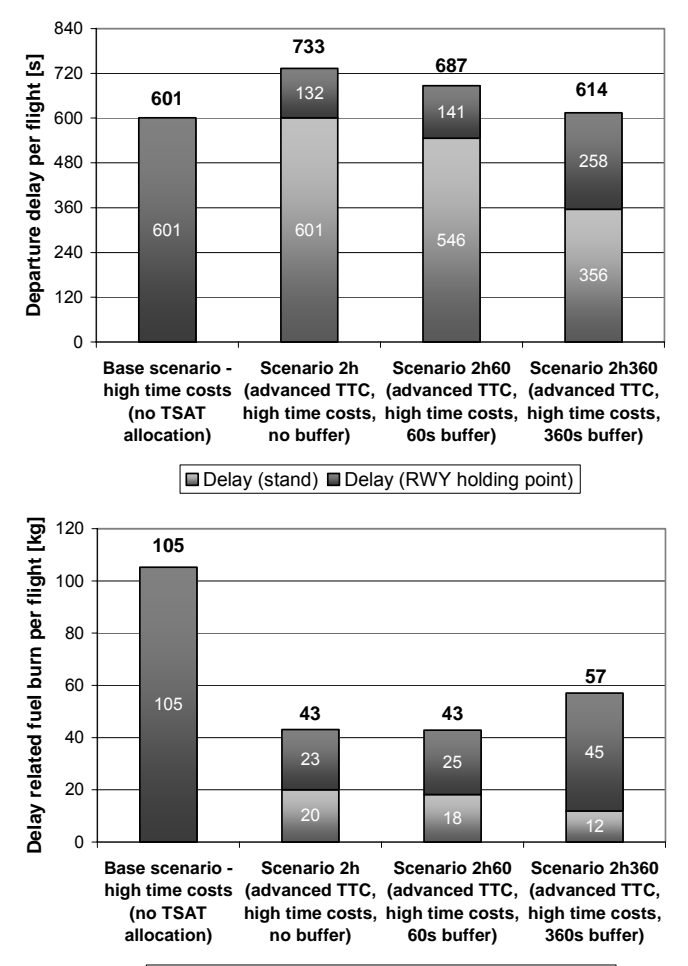

$\square$ Fuel Burn (stand) $\square$ Fuel Burn (RWY holding point)

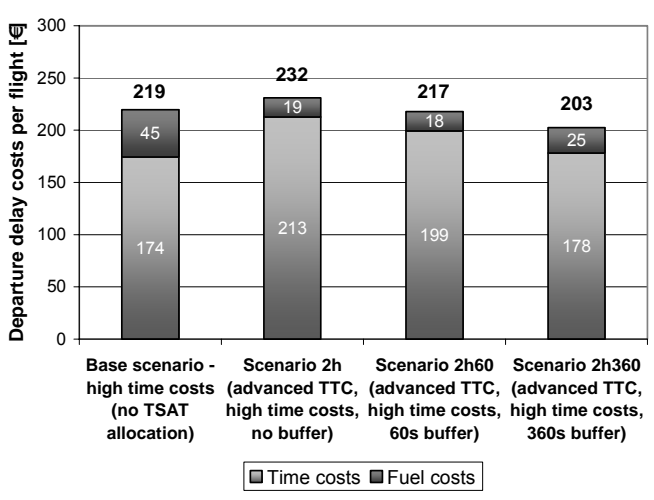

Figure 6. Impact of TSAT allocation strategies using different time buffers

\section{Conclusions and Outlook}

With regard to the presented results the following general conclusions can be drawn:

(1) Advanced taxi time calculation contributes to a reduction of delays, fuel burn, $\mathrm{CO}_{2}$ emissions and costs for airspace users compared to the use of static look-up tables.

(2) However, the shifting of delays from the runway holding positions to the stand does not necessarily contribute to efficiency improvements. The application of an unsuitable pre-departure sequencing strategy may lead to negative effects despite the use of advanced taxi time calculation.

(3) Pre-departure sequencing strategy must be chosen cautiously in dependence on the fuel and time costs for airlines as well the amount of departure delay that has to be absorbed. Using the formula TSAT = TTOT - EXOT as the general principle is not recommended.

Further research is required in view of the contributions of advanced taxi time calculation to improved gate and stand management. Thereby, the aim of taxi time calculation is to close the information gap between the inbound and outbound leg of an aircraft. For instance, better estimation of on-block times in dependence of runway allocation scenarios can contribute to the avoidance of stand occupancies in case of early inbound flights. This is achieved by using taxi time predictions to translate runway-related information (estimated landing time, ELDT) into stand-related information (estimated in-block time) as well as the other way round 
(target off-block time, TOBT into estimated take-off time). Additionally, taxi time calculation serves as an important link between arrival, departure and turnaround management systems (AMAN, DMAN, TMAN) in view of the sequence optimisation at the stands and the runway system. It is assumed that high accuracy of input times (ELDT, TOBT) alone does not to bring the desired benefits of sequencing criteria if taxi time durations with insufficient accuracy are used to compute target times. Hence, further research to gain a better understanding of the contribution of advanced taxi time calculation to the sequencing optimisation processes is recommended. This would require the integration of the pre-departure and the arrival/departure sequencing tools that were not available within this study and are still under development at a few airports. However, the fact that taxi times are used twice, both for the TSAT allocation for departures as presented within this paper and as input data for sequence optimisation in the context of arrival, departure and turnaround management, again emphasizes the relevance of the accuracy improvements provided by the advanced taxi time calculation.

\section{References}

1 ACI, Eurocontrol, IATA: “Airport CDM Implementation - The Manual”, Version 3, 2008.

2 ICAO: "Doc 9830 - Advanced Surface Movement Guidance and Control Systems (A-SMGCS) Manual", First Edition, 2004.

${ }^{3}$ Fricke, H., Schultz, M.: "Delay Impacts onto Turnaround Performance - Optimal Time Buffering for Minimizing Delay Propagation", in Proceedings of the USA/Europe Air Traffic Management Research and Development Seminar 2009.

${ }^{4}$ Oreschko, B., Schultz, M., Fricke, H.: "Significant Turnaround Process Variations due to Airport Characteristics", Air Transport and Operations Symposium 2010, in press.

${ }^{5}$ Pina, P., de Pablo, J. M.: "Benefits obtained from the Estimation and Distribution of Realistic Taxi Times", in Proceedings of the USA/Europe Air Traffic Management Research and Development Seminar 2005.

${ }^{6}$ Böhme, D.: „Tactical Departure Management with the EUROCONTROL / DLR DMAN”, in Proceedings of the USA/Europe Air Traffic Management Research and Development Seminar 2005.

${ }^{7}$ Hatzack, W.: "Operational Impact of Predeparture Sequencing", Presentation at the AGIFORS Airline Operations Conference 2005.

8 ATRiCS Advanced Traffic Solutions GmbH \& Co. KG: “ATRiCS SMAN VTTC”, Freiburg, 2007.

${ }^{9}$ Legge, J., Levy, B.: "Departure taxi time predictions using ASDE-X Surveillance Data", in Proceedings of the International Congress of the Aeronautical Sciences 2008.

${ }^{10}$ Balakrishna, P., Ganesan, R., Sherry, L.: “Application of Reinforcement Learning Algorithms for Predicting Taxi-out Times", in Proceedings of the USA/Europe Air Traffic Management Research and Development Seminar 2009.

${ }^{11}$ ICAO: „Doc 9854 - Global Air Traffic Management Operational Concept“, First Edition, 2005. 